\title{
Therapeutic Drug Monitoring
}

\section{A Population Pharmacokinetic Modeling and Simulation Study of Posaconazole Oral Suspension in Immunocompromised Pediatric Patients: A Short Communication

\author{
--Manuscript Draft--
}

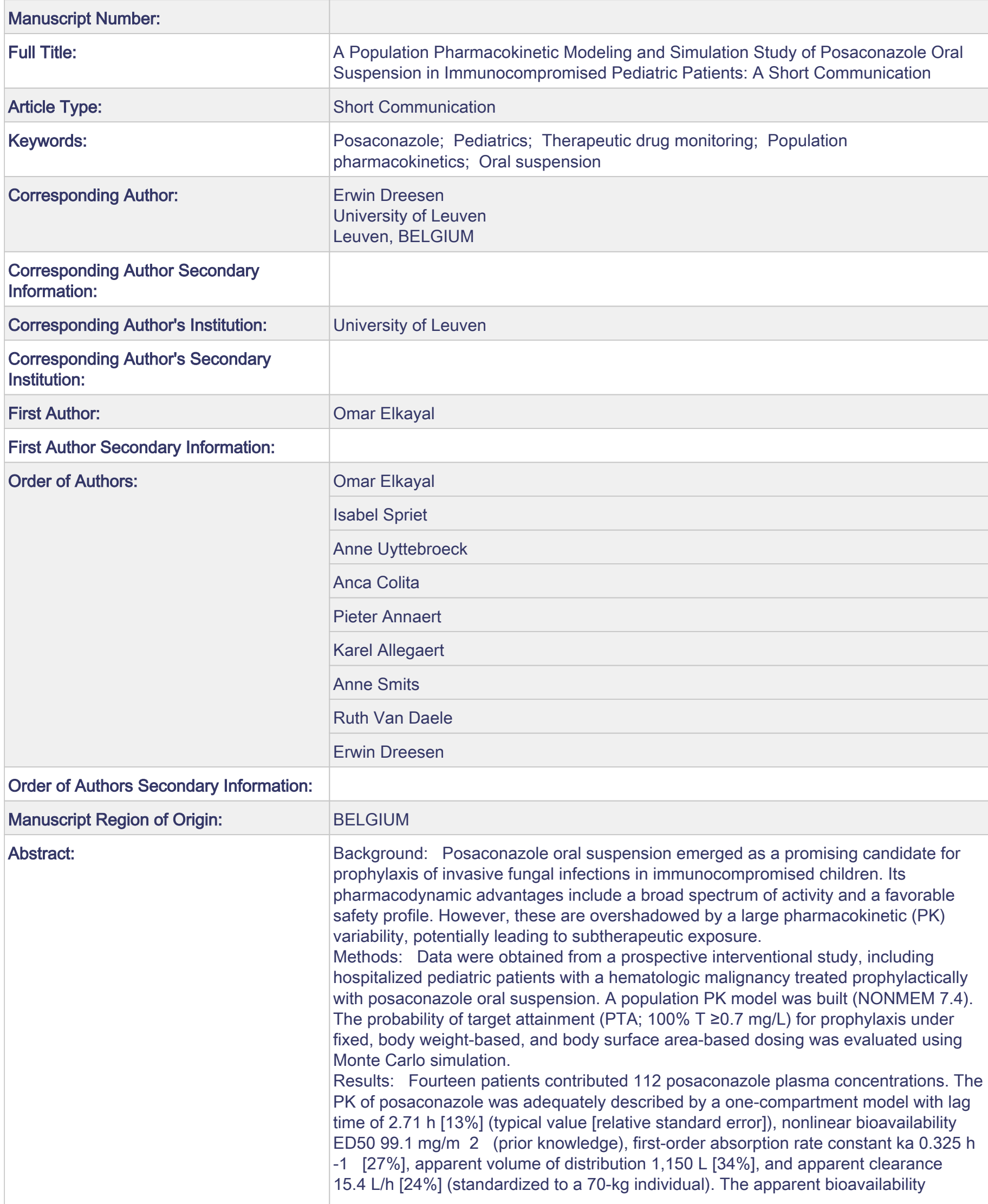


decreased in the presence of diarrhea and co-administration of a proton pump inhibitor (PPI). The unexplained interindividual variability in posaconazole PK remained large ( $86.4 \%$ coefficient of variation). The PTA was below $85 \%$, irrespective of the simulated dosing strategy. Patients without diarrhea and not receiving a PPI had the highest PTA, approaching $85 \%$ under fixed $300 \mathrm{mg}$ dosing four times a day.

Conclusion: Therapeutic drug monitoring is recommended during prophylactic posaconazole therapy in immunocompromised pediatric patients. Large scale comparative studies are urged to characterize the PK variability between the different posaconazole formulations in this patient population.

Suggested Reviewers:

Opposed Reviewers: 


\title{
KULEUVEN
}

\section{Subject: Submission of a Short Communication}

\author{
Dear Editor of Therapeutic Drug Monitoring
}

We hereby submit our manuscript entitled "A Population Pharmacokinetic Modeling and Simulation Study of Posaconazole Oral Suspension in Immunocompromised Pediatric Patients: A Short Communication” by Elkayal et al.

In our manuscript, we report the development of a population pharmacokinetic model of posaconazole oral suspension therapy in immunocompromised children based on data from a rich sampling study (112 posaconazole plasma concentrations contributed by 14 patients). Our analyses provided a more detailed insight into posaconazole pharmacokinetics, more specifically the absorption phase. We performed a simulation study to evaluate prophylaxis target attainment $(100 \% \mathrm{~T} \geq 0.7 \mathrm{mg} / \mathrm{L})$ under fixed, body weight-based, and body surface area-based dosing. The simulated probability of target attainment was below $85 \%$, irrespective of the dosing strategy. Patients without diarrhea not receiving a PPI had the highest probability of target attainment, approaching $85 \%$ under fixed $300 \mathrm{mg}$ dosing four times a day. Dose adjustments based on body weight or body surface area did not improve target attainment compared to fixed dosing. Low target attainment, wide interpatient pharmacokinetic variability, and a maximum desired exposure target of $3.75 \mathrm{mg} / \mathrm{L}$ (based on the $90^{\text {th }}$ percentile of exposure in Cornely et al.) demand the implementation of therapeutic drug monitoring in clinical practice. In case of underexposure on $300 \mathrm{mg}$ four times a day, the tablet or intravenous formulations may be considered. However, we believe that it is essential to further invest in dose optimization of the oral suspension in this specific off-label (and thus non-reimbursed) setting as both the delayed-release tablet and the intravenous formulation are very expensive.

As Therapeutic Drug Monitoring is known to be a leading journal for publishing clinical pharmacology research, including population pharmacokinetic modeling and simulation work, we believe our findings would appeal to the readers of Therapeutic Drug Monitoring.

All authors have read and approved the manuscript and agree with its submission to Therapeutic Drug Monitoring. The author contributions, the sources of funding, and the conflicts of interest of all authors are explicitly mentioned in the manuscript. We confirm that this manuscript has not been published elsewhere and is not under consideration by another journal.

We hope that our paper will be considered for publication in Therapeutic Drug Monitoring.

Yours sincerely, on behalf of all authors

\section{Cruin Dreesen}

\section{Erwin Dreesen}

FWO Postdoctoral Research Fellow Pharmacometrics

Clinical Pharmacology and Pharmacotherapy Unit

Department of Pharmaceutical and Pharmacological Sciences

KU Leuven, O\&N II Herestraat 49 - box 820, 3000 Leuven, Belgium, +32 16372753

Uppsala Pharmacometrics Research Group

Department of Pharmacy

Uppsala University, Biomedicinskt centrum BMC, Husargatan 3 - box 591, 75124 Uppsala, Sweden 
A Population Pharmacokinetic Modeling and Simulation Study of Posaconazole Oral Suspension in Immunocompromised Pediatric Patients

\author{
A Short Communication
}

Omar Elkayal ${ }^{1}$, MSc; Isabel Spriet ${ }^{1,2}$, PharmD, PhD; Anne Uyttebroeck ${ }^{3,4}, \mathrm{MD}, \mathrm{PhD}$; Anca Colita ${ }^{5,6}$, MD, PhD; Pieter Annaert ${ }^{1}$, PharmD, PhD; Karel Allegaert ${ }^{1,7}$, MD, PhD; Anne Smits ${ }^{7,8}$, MD, PhD; Ruth Van Daele ${ }^{1,2}$, PharmD; Erwin Dreesen ${ }^{1,9}$, PharmD, PhD

1 Department of Pharmaceutical and Pharmacological Sciences, KU Leuven, Leuven, Belgium

2 Pharmacy Department, University Hospitals Leuven, Leuven, Belgium

3 Department of Oncology, KU Leuven, Leuven, Belgium

4 Pediatric Oncology and Hematology, University Hospitals Leuven, Leuven, Belgium

5 Department of Pediatrics, Fundeni Clinical Institute, Bucharest, Romania

6 Department of Pediatrics, Carol Davila University of Medicine and Pharmacy, Bucharest, Romania

7 Department of Development and Regeneration, KU Leuven, Leuven, Belgium

8 Neonatal Intensive Care Unit, University Hospitals Leuven, Leuven, Belgium

9 Department of Pharmacy, Uppsala University, Uppsala, Sweden

Corresponding author: Erwin Dreesen

O\&N II Herestraat 49 - box 820

3000 Leuven, Belgium

Tel.: +3216372753

Email: erwin.dreesen@kuleuven.be

ORCID:

Omar Elkayal

Isabel Spriet

Anne Uyttebroeck

Anca Colita

Pieter Annaert https://orcid.org/0000-0001-5708-9497

https://orcid.org/0000-0001-6342-0676

https://orcid.org/0000-0001-5644-424X

https://orcid.org/0000-0001-6031-819

https://orcid.org/0000-0003-3525-7351 
Karel Allegaert

Anne Smits

Ruth Van Daele

Erwin Dreesen https://orcid.org/0000-0001-9921-5105

https://orcid.org/0000-0002-0710-6698

https://orcid.org/0000-0002-8353-2103

https://orcid.org/0000-0002-0785-2930

Source of Funding: $\quad$ IS is funded by the Clinical Research Fund of the University Hospitals Leuven. AS research activities are supported by the Clinical Research and Education Council of the University Hospitals Leuven. ED is a postdoctoral research fellow of the Research Foundation - Flanders (FWO), Belgium (grant number 12X9420N).

Conflicts of Interest: IS received consultancy support and lecture fees from argenx, Pfizer, Merck, and Cidara. PA is co-founder and co-owner of BioNotus (Niel, Belgium). RVD has received travel support from Pfizer, Inc., and Gilead Sciences. ED received consultancy fees from argenx and Janssen (all honoraria/fees paid to the University). For the remaining authors, none were declared.
Word count:
2,338

Table/Figure count: $\quad 1 / 1$

Number of references: 30 


\section{Abstract}

Background: Posaconazole oral suspension emerged as a promising candidate for prophylaxis of invasive fungal infections in immunocompromised children. Its pharmacodynamic advantages include a broad spectrum of activity and a favorable safety profile. However, these are overshadowed by a large pharmacokinetic (PK) variability, potentially leading to subtherapeutic exposure.

Methods: Data were obtained from a prospective interventional study, including hospitalized pediatric patients with a hematologic malignancy treated prophylactically with posaconazole oral suspension. A population PK model was built (NONMEM 7.4). The probability of target attainment (PTA; $100 \% \mathrm{~T} \geq 0.7 \mathrm{mg} / \mathrm{L}$ ) for prophylaxis under fixed, body weight-based, and body surface area-based dosing was evaluated using Monte Carlo simulation.

Results: Fourteen patients contributed 112 posaconazole plasma concentrations. The PK of posaconazole was adequately described by a one-compartment model with lag time of $2.71 \mathrm{~h} \mathrm{[13 \% ]}$ (typical value [relative standard error]), nonlinear bioavailability $\mathrm{ED}_{50} 99.1 \mathrm{mg} / \mathrm{m}^{2}$ (prior knowledge), first-order absorption rate constant $\mathrm{k}_{\mathrm{a}} 0.325 \mathrm{~h}^{-1}$ [27\%], apparent volume of distribution 1,150 L [34\%], and apparent clearance $15.4 \mathrm{~L} / \mathrm{h}$ [24\%] (standardized to a 70-kg individual). The apparent bioavailability decreased in the presence of diarrhea and co-administration of a proton pump inhibitor (PPI). The unexplained interindividual variability in posaconazole PK remained large $(86.4 \%$ coefficient of variation). The PTA was below $85 \%$, irrespective of the simulated dosing strategy. Patients without diarrhea and not receiving a PPI had the highest PTA, approaching $85 \%$ under fixed $300 \mathrm{mg}$ dosing four times a day.

Conclusion: Therapeutic drug monitoring is recommended during prophylactic posaconazole therapy in immunocompromised pediatric patients. Large scale comparative studies are urged to characterize the PK variability between the different posaconazole formulations in this patient population.

Key Words: Posaconazole; Pediatrics; Therapeutic drug monitoring; Population pharmacokinetics; Oral suspension 


\section{Introduction}

Posaconazole is often used in the prevention and treatment of invasive fungal infections (IFIs) in pediatric patients with graft-versus-host disease after stem cell transplantation and febrile neutropenia due to its broad antifungal activity, its favorable safety profile, and the lack of more appropriate alternatives. ${ }^{1-4}$ Since posaconazole is prescribed off-label in this setting, the oral suspension is most commonly used given its lower cost and ease of administration as compared to the delayed-release tablet and intravenous formulation. The challenge facing posaconazole suspension is the large pharmacokinetic (PK) variability between patients, particularly in terms of absorption, often leading to suboptimal exposure. ${ }^{1,5}$ It has been shown that in children, the standard (adult) dosing fails to assure the therapeutic posaconazole trough concentrations at steady-state of $0.7 \mathrm{mg} / \mathrm{L}$ and $1.0 \mathrm{mg} / \mathrm{L}$ for prophylaxis and treatment of IFIs, respectively. ${ }^{4}$ Therefore, therapeutic drug monitoring (TDM) has been used as a tool to quantify posaconazole exposure and to adapt dosing accordingly. ${ }^{6}$

To date, no pediatric posaconazole dosing regimen has been agreed upon for none of the different posaconazole formulations. Some clinical centers have published their experience with TDM of posaconazole in pediatric patients. ${ }^{7-11}$ The large variability in exposure and the negative impact of diarrhea and concomitant proton-pump inhibition (PPI) was established in these studies. Vanstraelen et $a l$. proposed dosing in pediatric patients based on the body surface area (BSA) using a dosage regimen of $120 \mathrm{mg} / \mathrm{m}^{2}$ three times a day. ${ }^{12}$

A population PK (popPK) model may provide insight into sources of variability in exposure, can be used to support dose-finding simulations, and may be used to facilitate model-based TDM if warranted. One posaconazole popPK model has previously been published by Boonsathorn et al., in which concomitant PPI and diarrhea explained variability in the bioavailability of the posaconazole oral suspension. ${ }^{13}$ Based on their model, the authors suggested several dosing strategies for posaconazole in children. However, since their model was based on sparse TDM sampling, the absorption parameters could not be estimated. Also, an external evaluation of their model and dosing suggestions is awaited. Our aim was to develop a popPK model based on rich sampling, leading to a more detailed insight into the PK of the posaconazole oral suspension. We performed Monte Carlo simulations to evaluate target attainment under fixed, body weight-based, and BSA-based dosing. Finally, the predictive performance of both our newly developed model and the previously published was compared. 


\section{Patients and Data Collection}

Data were obtained from a prospective study by Vanstraelen et $a .^{12}$ In this study, 14 immunocompromised children aged 13 years or younger with a hematological malignancy were treated prophylactically with the posaconazole oral suspension (Noxafil, Merck, Brussels, Belgium) at a dose of $120 \mathrm{mg} / \mathrm{m}^{2}$ three times daily, taken with a meal. ${ }^{12}$ Eight blood samples were collected from each patient during one dosing interval at steady-state (after at least eight days of treatment). The study protocol was approved by the local Ethics Committee (NCT02372357). Written informed consent was obtained from each child's relative. The study was conducted in accordance with the Declaration of Helsinki.

\section{Analytical Procedures}

All heparinized blood samples were centrifuged immediately after sampling (10 minutes, $\left.1,500 \mathrm{~g}, 20^{\circ} \mathrm{C}\right)$, plasma was stored at $-20^{\circ} \mathrm{C}$ until further analysis. ${ }^{12}$ Total posaconazole plasma concentrations were measured using high-performance liquid chromatography followed by fluorescence detection. ${ }^{12,14}$

\section{Population Pharmacokinetic Modeling}

Software

Dataset formatting and exploration were performed using R (version 3.5.1, R Core Team, Vienna, Austria) using custom scripts based on packages including dplyr and ggplot2. ${ }^{15,16}$ PopPK analysis was performed using NONMEM (version 7.4; ICON Development Solutions, Gaithersburg, Maryland, USA). ${ }^{17}$

\section{Model Development}

126 Parameter estimation was performed using first-order conditional estimation with interaction (FOCE-I) and differential equation solver ADVAN 6. A structural base popPK model was developed. Different structural models with varying numbers of compartments, and linear and nonlinear absorption and elimination processes were explored. Allometric scaling was tested with exponents of $0.75,1$, and 0.25 on clearance $(\mathrm{CL} / \mathrm{F})$, volume of distribution $\left(\mathrm{V}_{\mathrm{d}} / \mathrm{F}\right)$, and absorption rate constant $\left(\mathrm{k}_{\mathrm{a}} / \mathrm{F}\right)$, respectively. ${ }^{18}$

131 Since the posaconazole suspension has been shown to demonstrate nonlinear absorption ${ }^{19}$, the bioavailability $\mathrm{F}$ of the oral suspension was described as 
134 where $\mathrm{D}$ is the dose in $\mathrm{mg} / \mathrm{m}^{2} \mathrm{BSA}$ and $\mathrm{ED}_{50}$ is the estimated dose in $\mathrm{mg} / \mathrm{m}^{2}$ that yields a $50 \%$ decrease

135 in relative bioavailability of the suspension. $\mathrm{ED}_{50}$ was fixed to $99 \mathrm{mg} / \mathrm{m}^{2}$ based on previous literature. ${ }^{13}$

136 Individual PK parameters were assumed to be log-normally distributed, which was achieved using an exponential formula, e.g., the clearance (CL) parametrization followed this equation:

$$
\mathrm{CL}_{i}=\mathrm{TVCL} \times \exp \left(\eta_{i}\right) \text { with } \eta_{i} \propto N\left(0, \omega^{2}\right)
$$

139

with $\mathrm{CL}_{i}$ the clearance of subject $i$, TVCL the typical population value of the clearance, and $\eta_{i}$ the individual patient's deviation from the typical value.

The base model was selected based on objective function value (OFV) comparisons (difference $\geq 3.84$ points; $\mathrm{P} \leq 0.050$ ), plausibility and precision of parameter estimates, and goodness-of-fit plots. A final model, including covariate effects, was built through a two-way stepwise covariate modeling (SCM) procedure (forward inclusion $\alpha=0.010$; backward elimination $\alpha=0.001$ ). The tested covariates were weight, co-treatment with a PPI, and presence of diarrhea.

\section{Final Model Evaluation}

The final model was evaluated using a prediction-corrected visual predictive check (pcVPC; $n=1,000$ simulated replicates of the original dataset). Furthermore, bootstrapping was performed to obtain nonparametric estimates of uncertainty in parameter estimates ( $n=2,000$ bootstraps).

\section{Dosing Simulations}

Monte Carlo simulations were performed using the final popPK model to evaluate different posaconazole dosing strategies that have recently been suggested in literature. ${ }^{5,6,12,13}$ In each dosing scenario, PK profiles of 1,000 patients were simulated at steady-state, and the probability of target attainment (PTA) for prophylaxis (total posaconazole concentration $100 \% \mathrm{~T} \geq 0.7 \mathrm{mg} / \mathrm{L}$ ) was calculated. Continuous covariates were randomly sampled from a uniform distribution within the range of the original data set. Combinations of binary covariates were assigned in equal proportions.

\section{Predictive Performance Assessment}

External (Boonsathorn et al. ${ }^{13}$ ) and internal predictive performance were assessed. ${ }^{20}$ Model-predicted and observed individual concentrations were compared by calculating the relative bias (rBias) and relative root mean squared error (rRMSE). ${ }^{20}$

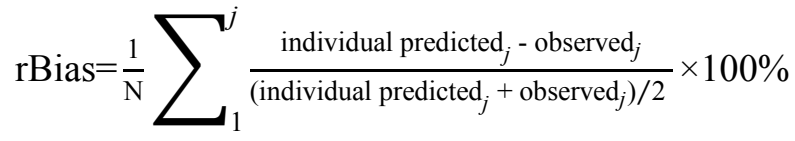




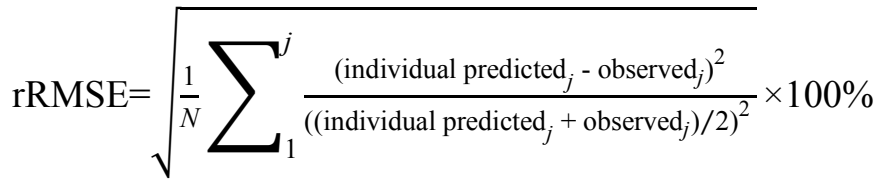

163 with $N$ the total number of observed posaconazole concentrations. 


\section{Results}

\section{Patient Characteristics}

A total of 14 patients (median [IQR] age 7 [5-8] years, body weight 20.5 [16.9-21.9] kg) contributed 112 plasma samples to the analysis. Eight out of 14 patients (57\%) achieved the prophylaxis target. Six patients were suffering from diarrhea and ten patients were concomitantly treated with a PPI.

\section{Population Pharmacokinetic Model}

Base Model

A one-compartment model with a lag time, nonlinear relative bioavailability, first-order absorption, and linear elimination best described the data (Table 1). Allometric scaling was implemented. The unexplained interindividual variability in the apparent clearance was estimated at $87.9 \%$ [25\%] (coefficient of variation [relative standard error]). A proportional error model was used to describe the unexplained residual variability.

\section{Final Model}

Inclusion of the effects of diarrhea and PPI comedication on the relative bioavailability did not result in a significant drop in OFV ( $\triangle \mathrm{OFV}=-1.1$ and -1.2 , respectively). Nevertheless, inclusion of these effects on F using fixed parameter values derived from literature improved the accuracy of the parameter estimates (Table 1). ${ }^{13}$

\section{Model Evaluation}

Goodness-of-fit plots confirmed that the final model adequately described the observed data (not shown). The pcVPC showed a good agreement between the observed data and model simulations (Figure 1A). Median values of the nonparametric bootstrap were in good agreement with the FOCE-I point estimates (Table 1).

\section{Dosing Simulations}

The predicted PTA under fixed, body weight-based, and BSA-based dosing are given in Figure 1B-D. Posaconazole dose adjustments based on body weight or BSA did not improve the PTA as compared to fixed dosing. Posaconazole trough concentrations and thus the PTA were negatively affected by the presence of diarrhea and PPI co-administration. The highest PTA (85\%) was attained for the dosage regimens of $300 \mathrm{mg}$ four times a day and $24 \mathrm{mg} / \mathrm{kg}$ four times a day in patients not suffering from diarrhea and not receiving PPI co-administration. Due to saturable absorption, the PTA plateaued when increasing the dose above $300 \mathrm{mg}$ and $24 \mathrm{mg} / \mathrm{kg}$. 


\section{Predictive Performance Assessment}

195 Predictive performance assessment demonstrated that the previously published model underpredicted

196 posaconazole exposure in our study data (rBias $-9.8 \%$, pcVPC not shown). Our model not only predicted

197 the observed posaconazole concentrations with negligible bias $(0.4 \%)$ but also with higher precision

198 (rRMSE 10.7\%) as compared to the literature model (25.6\%).

199 Using the patient's first pre-dose concentration in a Bayesian forecasting scenario, both models

200 predicted the second pre-dose concentration with acceptable bias $(1.2 \%$ and $-4.9 \%$ using our study

201 model and the literature model, respectively). However, our model performed better in terms of 202 precision $(23.2 \%)$ than the literature model (41.8\%). 
We performed a popPK modeling and simulation study of posaconazole oral suspension therapy in immunocompromised children. Our analyses provided a more detailed insight into posaconazole PK and resulted in dosing recommendations in this specific setting. Furthermore, we compared the predictive performance of our model and a previously published model.

The one-compartment model with lag time and inter-individual variability on clearance best described the PK of posaconazole. The inclusion of a lag time is the main structural improvement compared to the previously published posaconazole popPK model ${ }^{13}$ This additional PK feature is attributed to the insight that the rich sampling scheme provided to our model. The capture of a lag time is in line with the finding from Dolton et al. in an adult population. ${ }^{21}$ Rich sampling also enabled us to estimate the first-order absorption rate constant, which needed to be fixed in the previously published model that was built using sparse TDM data. The difference in absorption rate constant in our model $\left(0.325 \mathrm{~h}^{-1}\right)$ and the previously published value $\left(0.197 \mathrm{~h}^{-1}\right)$ is likely to be explained by the lack of a lag time in the latter. ${ }^{22}$ While the apparent clearance in our model is in line with the previously published value, the volume of distribution in our model $(1,150 \mathrm{~L}$; standardized to a $70-\mathrm{kg}$ patient $)$ is closer to the known high volume of distribution of posaconazole. ${ }^{23}$

Previously published popPK models of posaconazole in both adults and children included the effects of diarrhea on bioavailability. ${ }^{13,21,24,25}$ Diarrhea is common in patients suffering from graft-versus-host disease, critically ill patients, and post-chemotherapy patients. In our cohort, six out of 14 patients had diarrhea. Since our study was underpowered to identify the effect of diarrhea, it was added to the model based on prior knowledge, resulting in a 33\% decrease in the apparent bioavailability of the oral posaconazole suspension. Similarly, the co-administration of a PPI is common practice in this setting and has been shown to affect posaconazole bioavailability negatively (reduced dissolution). ${ }^{13,14,26-28}$ Likewise, the impact of concomitant PPI intake was implemented in our model, making the apparent bioavailability decrease with $42 \%$. Since the Boonsathorn et al. model is based on a large patient cohort, we feel confident putting our trust in their parameter estimates. Due to the limited number of patients in our study, we were cautious when testing more covariates like age and concomitant use of an $\mathrm{H}_{2}$-receptor antagonist to avoid overfitting the model. Future studies with higher patient numbers may evaluate these and other potential covariate effects like concomitant food intake and vomiting. All patients in our cohort received posaconazole with a high-fat meal/supplement and vomiting was not reported.

Our simulation results showed that dose adjustment based on body weight or BSA did not improve target attainment compared to fixed dosing. Irrespective of the dose and dosing interval, the PTA did not exceed $85 \%$. Due to the saturable nature of posaconazole absorption, the PTA plateaued around $300 \mathrm{mg}$, making further dose increases futile. ${ }^{13}$ On the other hand, our simulations showed that dose fractionation 
237 effectively increases the PTA, although dosing more than four times a day may be impractical. A fixed

$238300 \mathrm{mg}$ dose of posaconazole administered four times a day resulted in the highest predicted mean PTA,

239 approaching $85 \%$. Low PTA, wide interpatient variability, and a maximum desired exposure target of

$2403.75 \mathrm{mg} / \mathrm{L}$ (based on the $90^{\text {th }}$ percentile of exposure in Cornely et al. ${ }^{29}$ ) demand the implementation of

241 TDM in clinical practice. In case of underexposure on $300 \mathrm{mg}$ four times a day, the tablet or intravenous

242 formulations may be considered. Unfortunately, the difficulty of swallowing and high cost may

243 undermine their second-line place.

244 Considering a role for TDM during posaconazole oral suspension therapy, we evaluated the predictive

245 performance of our model (internal validation) and the literature model (external validation) in a model-

246 informed precision dosing (MIPD) context. Bayesian forecasting of the next posaconazole trough

247 concentration based on the current trough concentration showed acceptable bias $(<5 \%)$ when using

248 either model. However, the published model had an unacceptably high rRMSE (41.8\%), while our model

249 was still within the arbitrarily accepted range $(<25 \%)$. Future qualification of our model for MIPD using

250 multiple external datasets may be needed to support generalizability of our findings. ${ }^{30}$

251 Our study has several strengths. Firstly, our dataset included rich sampling, which enabled us to capture

252 the lag time and absorption rate constant accurately. Secondly, our data had very consistent reporting

253 with no missing samples. Some limitations, however, remain. Firstly, the low number of patients

254 resulted in an artificially inflated interindividual variability, thereby making the covariate search

255 unfruitful. Still, model diagnosis showed an adequate model fit. Secondly, all included patients were

256 treated with posaconazole oral suspension, preventing us from comparing different dosing forms.

257 However, we believe that it is essential to further invest in dose optimization of the oral suspension in

258 this specific off-label (and thus non-reimbursed) setting as both the delayed-release tablet and the

259 intravenous formulation are very expensive. Population modeling of a larger dataset, including rich

260 sampling and including patients treated with the different formulations, is needed to allow well-informed

261 dose-finding for immunocompromised children. 


\section{Conclusion}

263 Our modeling and simulation study illustrates the challenges related to the attainment of adequate 264 prophylactic exposure when treating immunocompromised children with the posaconazole oral 265 suspension. Poor target attainment, especially in children with diarrhea and PPI co-treatment, wide 266 interpatient PK variability, and a relatively narrow therapeutic window $(0.7-3.75 \mathrm{mg} / \mathrm{L})$, support a role 267 for TDM during treatment with the posaconazole oral suspension. 


\section{References}

1. Cornely OA, Maertens J, Winston DJ, et al. Posaconazole vs. Fluconazole or Itraconazole Prophylaxis in Patients with Neutropenia. N Engl J Med. 2007;356(4):348-359.

2. Li Y, Theuretzbacher U, Clancy CJ, Nguyen MH, Derendorf $\mathrm{H}$. Pharmacokinetic/Pharmacodynamic Profile of Posaconazole. Clin Pharmacokinet. 2010;49(6):379-396.

3. Tarani L, Costantino F, Notheis G, et al. Long-term posaconazole treatment and follow-up of rhino-orbital-cerebral mucormycosis in a diabetic girl. Pediatr Diabetes. 2009;10(4):289-293.

4. Ashbee HR, Barnes RA, Johnson EM, Richardson MD, Gorton R, Hope WW. Therapeutic drug monitoring (TDM) of antifungal agents: guidelines from the British Society for Medical Mycology. J Antimicrob Chemother. 2014;69(5):1162-1176.

5. Vehreschild JJ, Müller C, Farowski F, et al. Factors influencing the pharmacokinetics of prophylactic posaconazole oral suspension in patients with acute myeloid leukemia or myelodysplastic syndrome. Eur J Clin Pharmacol. 2012;68(6):987-995.

6. Dolton MJ, Ray JE, Marriott D, McLachlan AJ. Posaconazole Exposure-Response Relationship: Evaluating the Utility of Therapeutic Drug Monitoring. Antimicrob Agents Chemother. 2012;56(6):2806-2813.

7. Bernardo VA, Cross SJ, Crews KR, et al. Posaconazole Therapeutic Drug Monitoring in Pediatrics and Young Adults with Cancer. Ann Pharmacother. 2013;47(0):976-983.

8. Girmenia C, Annino L, Mariotti B, et al. Posaconazole oral suspension primary prophylaxis in acute leukemia and allogeneic stem cell transplant patients: can it be used without measurement of plasma concentration? Med Mycol. 2016;54(5):445-458.

9. Jancel T, Shaw PA, Hallahan CW, et al. Therapeutic drug monitoring of posaconazole oral suspension in paediatric patients younger than 13 years of age: a retrospective analysis and literature review. J Clin Pharm Ther. 2017;42(1):75-79.

10. Vicenzi EB, Calore E, Decembrino N, et al. Posaconazole oral dose and plasma levels in pediatric hematology-oncology patients. Eur J Haematol. 2018;100(3):315-322.

11. Wass EN, Hernandez EA, Sierra CM. Comparison of the Efficacy of Posaconazole Delayed Release Tablets and Suspension in Pediatric Hematology/Oncology Patients. J Pediatr Pharmacol Ther. 2020;25(1):47-52.

12. Vanstraelen K, Colita A, Bica AM, et al. Pharmacokinetics of Posaconazole Oral Suspension in Children Dosed According to Body Surface Area: Pediatr Infect Dis J. 2016;35(2):183-188.

13. Boonsathorn S, Cheng I, Kloprogge F, et al. Clinical Pharmacokinetics and Dose Recommendations for Posaconazole in Infants and Children. Clin Pharmacokinet. 2019;58(1):5361.

14. Walravens J, Brouwers J, Spriet I, Tack J, Annaert P, Augustijns P. Effect of $\mathrm{pH}$ and Comedication on Gastrointestinal Absorption of Posaconazole. Clin Pharmacokinet. 2011;50(11):725-734.

15. Wickham H, François R, Henry L, Müller K, RStudio. Dplyr: A Grammar of Data Manipulation.; 2020. 
17. Beal S, Boeckmann L, Bauer R, Sheiner L. NONMEM User's Guides. (1989-2009).

18. Germovsek E, Barker CIS, Sharland M, Standing JF. Scaling clearance in paediatric pharmacokinetics: All models are wrong, which are useful? Br J Clin Pharmacol. 2017;83(4):777790.

19. Krishna G, Ma L, Martinho M, O'Mara E. Single-dose phase I study to evaluate the pharmacokinetics of posaconazole in new tablet and capsule formulations relative to oral suspension. Antimicrob Agents Chemother. 2012;56(8):4196-4201.

20. Sheiner LB, Beal SL. Some suggestions for measuring predictive performance. J Pharmacokinet Biopharm. 1981;9(4):503-512.

21. Dolton MJ, Brüggemann RJM, Burger DM, McLachlan AJ. Understanding Variability in Posaconazole Exposure Using an Integrated Population Pharmacokinetic Analysis. Antimicrob Agents Chemother. 2014;58(11):6879-6885.

22. Ezzet F, Wexler D, Courtney R, Krishna G, Lim J, Laughlin M. Oral Bioavailability of Posaconazole in Fasted Healthy Subjects. Clin Pharmacokinet. 2005;44(2):211-220.

23. Chen L, Krekels EHJ, Verweij PaulE, Buil JB, Knibbe CAJ, Brüggemann RJM. Pharmacokinetics and Pharmacodynamics of Posaconazole. Drugs. 2020;80(7):671-695.

24. Kohl V, Müller C, Cornely OA, et al. Factors Influencing Pharmacokinetics of Prophylactic Posaconazole in Patients Undergoing Allogeneic Stem Cell Transplantation. Antimicrob Agents Chemother. 2010;54(1):207-212.

25. AbuTarif MA, Krishna G, Statkevich P. Population pharmacokinetics of posaconazole in neutropenic patients receiving chemotherapy for acute myelogenous leukemia or myelodysplastic syndrome. Curr Med Res Opin. 2010;26(2):397-405.

26. Krishna G, Moton A, Ma L, Medlock MM, McLeod J. Pharmacokinetics and Absorption of Posaconazole Oral Suspension under Various Gastric Conditions in Healthy Volunteers. Antimicrob Agents Chemother. 2009;53(3):958-966.

27. Krishna G, AbuTarif M, Xuan F, Martinho M, Angulo D, Cornely OA. Pharmacokinetics of oral posaconazole in neutropenic patients receiving chemotherapy for acute myelogenous leukemia or myelodysplastic syndrome. Pharmacotherapy. 2008;28(10):1223-1232.

28. Alffenaar J-WC, van Assen S, van der Werf TS, Kosterink JGW, Uges DRA. Omeprazole Significantly Reduces Posaconazole Serum Trough Level. Clin Infect Dis. 2009;48(6):839-839.

29. Cornely OA, Duarte RF, Haider S, et al. Phase 3 pharmacokinetics and safety study of a posaconazole tablet formulation in patients at risk for invasive fungal disease. J Antimicrob Chemother. 2016;71(3):718-726.

30. Kantasiripitak W, Van Daele R, Gijsen M, Ferrante M, Spriet I, Dreesen E. Software Tools for Model-Informed Precision Dosing: How Well Do They Satisfy the Needs? Front Pharmacol. 2020;11:620. 


\section{Figure legends}

346 Figure 1. (A) Prediction-corrected visual predictive check of the final population pharmacokinetic

347 model. Open circles represent the observed prediction-corrected posaconazole concentrations. The black

348 solid and dash lines represent median and $2.5^{\text {th }}$ and $97.5^{\text {th }}$ percentiles of the prediction-corrected

349 observations, respectively. The shaded areas indicate $90 \%$ prediction-intervals of the median and $2.5^{\text {th }}$

350 and $97.5^{\text {th }}$ percentile of the simulated values. (B-D) The simulated probability of target attainment of the

$351100 \% \mathrm{~T} \geq 0.7 \mathrm{mg} / \mathrm{L}$ prophylaxis target at steady state for fixed dosing, weight-adjusted dosing, and body

352 surface area-adjusted dosing, respectively. b.i.d.: twice daily; PPI: proton pump inhibition; q.i.d: four

353 times daily; t.i.d.: three times daily. 


\section{Tables}

2 Table 1. Summary of the population pharmacokinetic model parameter estimates

\begin{tabular}{|c|c|c|c|c|c|c|c|}
\hline \multirow[t]{2}{*}{ Parameter } & \multicolumn{2}{|l|}{ Base model } & \multicolumn{3}{|l|}{ Final model } & \multicolumn{2}{|c|}{ Boonsathorn et al. ${ }^{13}$} \\
\hline & Estimate & $\begin{array}{l}\% R S E \\
\text { [\%shrinkage] }\end{array}$ & Estimate & $\begin{array}{l}\text { \%RSE } \\
\text { [\%shrinkage] }\end{array}$ & $\begin{array}{l}\text { Bootstrap median } \\
(95 \% \text { CI })\end{array}$ & Estimate & $\% R S E$ \\
\hline \multicolumn{8}{|l|}{ Typical values } \\
\hline $\mathrm{T}_{\mathrm{lag},} h$ & 2.79 & 15 & 2.71 & 13 & $2.79(0.8-3.979)$ & - & - \\
\hline $\mathrm{k}_{\mathrm{a}}, h^{-1}$ & 0.258 & 42 & 0.325 & 27 & $0.315(0.03-13.99)$ & 0.197 FIX & - \\
\hline $\mathrm{ED}_{50}, \mathrm{mg} / \mathrm{m}^{2}$ & 99.1 FIX & - & 99.1 FIX & - & 99.1 FIX & 99.1 & 44 \\
\hline $\mathrm{V} / \mathrm{F}, L$ & 1,410 & 43 & 1,150 & 34 & $1,036(50-3,973)$ & 201.7 & 38.8 \\
\hline $\mathrm{CL} / \mathrm{F}, L / h$ & 21.4 & 24 & 15.4 & 24 & $15.4(9.8-25.4)$ & 14.95 & 34.5 \\
\hline Diarrhea on bioavailability & - & - & -0.33 FIX & - & -0.33 FIX & -0.33 & 28 \\
\hline PPI on bioavailability & - & - & -0.42 FIX & - & -0.42 FIX & -0.42 & 14.9 \\
\hline \multicolumn{8}{|l|}{ Interindividual variability } \\
\hline on $\mathrm{CL} / \mathrm{F}, \% C V$ & 87.9 & $15.9[0]$ & 87.8 & $20[0.1]$ & $86.4(18.7-227.0)$ & 63 & 23.9 \\
\hline \multicolumn{8}{|l|}{ Residual variability } \\
\hline Proportional error, $\% C V$ & 16 & $8[6]$ & 11 & $8[6]$ & $0.01198(0.008-0.1597)$ & 47.29 & 0.2 \\
\hline Additive error, $m g / L$ & - & - & - & - & - & 0.02 & 82.7 \\
\hline
\end{tabular}

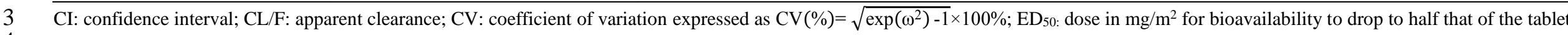

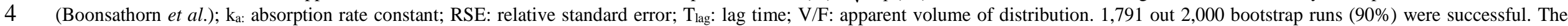
condition number was 69.51 . 


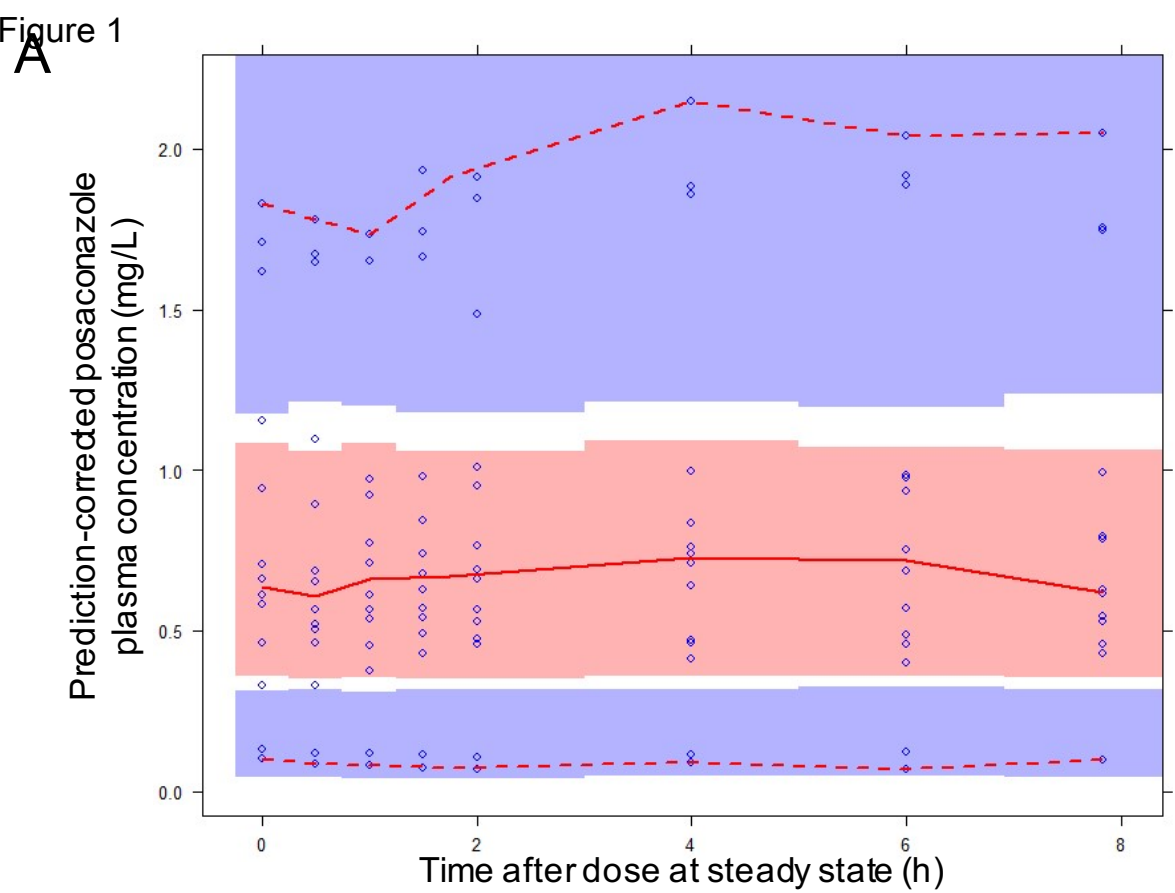

B

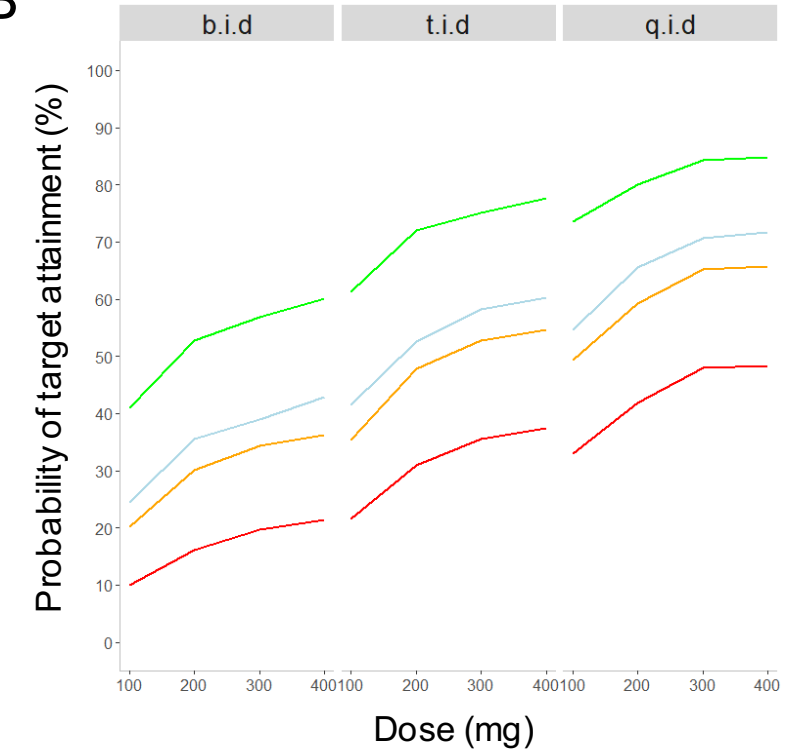

C

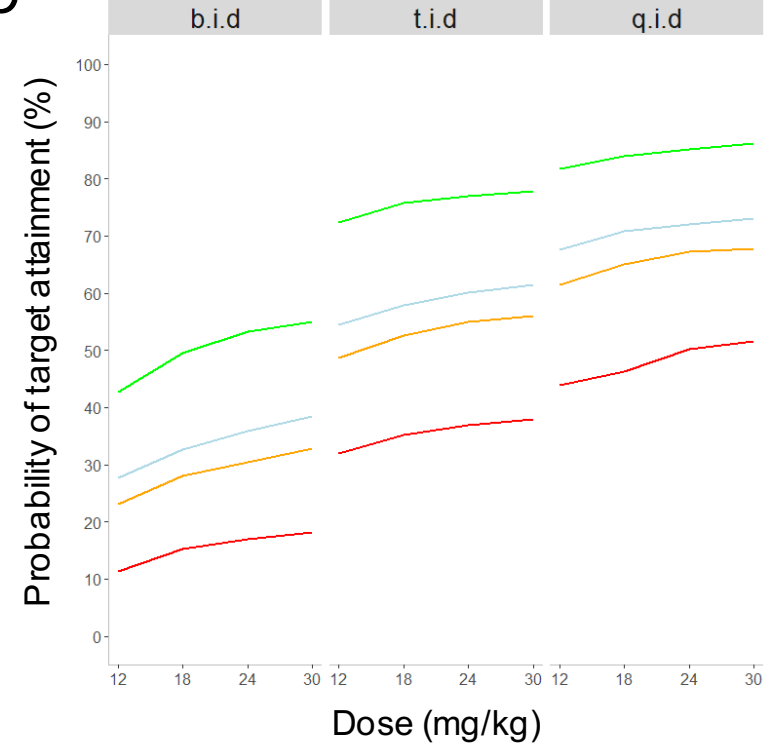

- No diarrhea, no PPI Diarrhea, no PPI

- No diarrhea, PPI

- Diarrhea, PPI

D

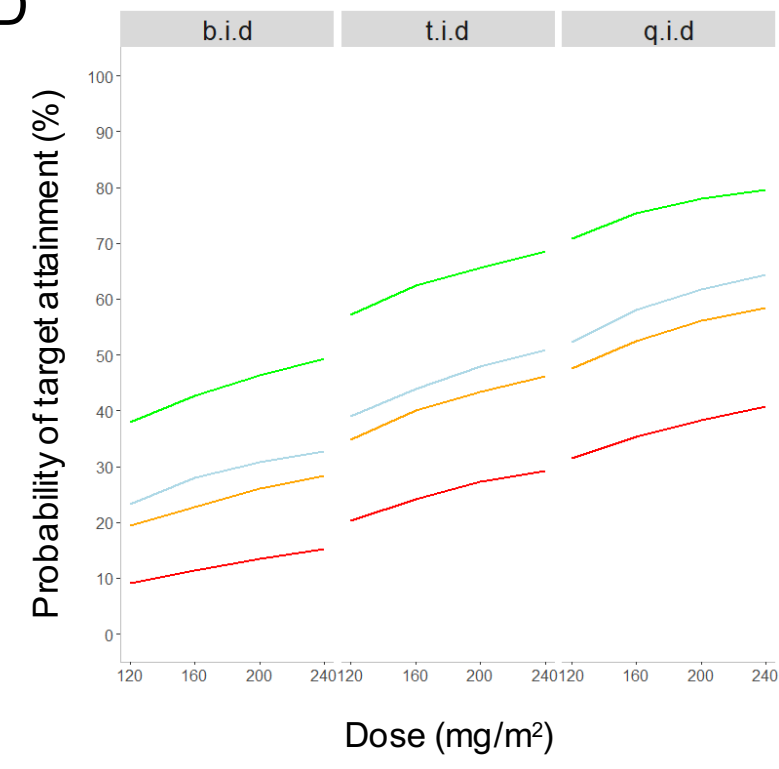


Click here to access/download Copyright Transfer and Disclosure Form coi_disclosure_AC.pdf 


\section{Click here to access/download Copyright Transfer and Disclosure Form \\ coi_disclosure_AS.pdf}


Click here to access/download Copyright Transfer and Disclosure Form coi_disclosure_AU.pdf 
Click here to access/download Copyright Transfer and Disclosure Form coi_disclosure_ED.pdf 
Click here to access/download Copyright Transfer and Disclosure Form coi_disclosure_IS.pdf 


\section{Click here to access/download \\ Copyright Transfer and Disclosure Form coi_disclosure_KA.pdf}


Click here to access/download Copyright Transfer and Disclosure Form coi_disclosure_OE.pdf 


\section{Click here to access/download \\ Copyright Transfer and Disclosure Form coi_disclosure_PA.pdf}


Click here to access/download Copyright Transfer and Disclosure Form coi_disclosure_RVD.pdf 